\title{
Contextual Modulation in Primary Visual Cortex of Macaques
}

\author{
Andrew F. Rossi, Robert Desimone, and Leslie G. Ungerleider \\ Laboratory of Brain and Cognition, National Institute of Mental Health, National Institutes of Health, Bethesda, \\ Maryland 20892
}

\begin{abstract}
Recent studies have suggested that V1 neurons extract figures from their backgrounds, in that they respond better to interior features of figures than to equivalent features of background stimuli. This is reportedly true even when the figure boundaries are distant from the borders of the classical receptive field (RF). To test the role of V1 neurons in figure-ground segregation, we recorded their responses to texture figures on texture backgrounds, centered on the RF. The texture elements of the figures remained identical across trials, and figure boundaries were defined by orientation differences between the elements in the background texture relative to elements in the figure. For nearly all neurons (98/102), responses to a large texture figure did not differ from the responses to a uniform-texture background. Although many neurons gave enhanced responses to texture boundaries, this occurred only when the boundaries were within or close to the RF borders. Similar effects were
\end{abstract}

found in V2. For neurons in V1, the limited spatial extent of the contextual modulation was not increased either at low stimulus contrast or when the animal was rewarded for detecting an orientation-defined figure. Thus, V1 neurons appear to signal texture boundaries rather than figures per se. Unexpectedly, many $\mathrm{V} 1$ neurons gave significant long-latency responses to texture stimuli located entirely outside the classical RF, up to $5^{\circ}$ from the RF border in some cases. However, these responses did not depend on the stimulus forming a figure that contained the RF. Although V1 neurons are influenced by stimuli outside the classical RF, they do not appear to segregate figures from ground.

Key words: figure-ground segregation; surface perception; striate cortex; visual perception; contextual modulation; nonclassical receptive field; texture segregation
The responses of $\mathrm{V} 1$ neurons to a stimulus in the receptive field (RF) are known to be influenced by stimuli outside the RF (Maffei and Fiorentini, 1976; Nelson and Frost, 1978; Allman et al., 1985; Gilbert and Wiesel, 1990). Thus, V1 neurons not only signal the presence of a stimulus in the RF but also convey information about the context in which the stimulus is presented. Contextual modulation in V1 has been interpreted as the neural substrate of a variety of psychophysical phenomena, such as perceptual pop-out (Knierim and Van Essen, 1992; Kastner et al., 1997), contour integration (Kapadia et al., 1995), surface perception (Rossi et al., 1996; MacEvoy et al., 1998), and figure-ground segregation (Lamme, 1995; Zipser et al., 1996; Lee et al., 1998).

The specific effects of extra-RF stimulation vary significantly depending on the configuration of the stimulus array. Several studies have tested the role of global context by measuring the response to a line segment in the $\mathrm{RF}$, embedded in an array of other line segments of the same or different orientation outside the RF (Knierim and Van Essen, 1992; Kastner et al., 1997; Li et al., 2000). The responses of most neurons to the line segment in the RF were suppressed by the surrounding array, and the degree of suppression varied according to the similarity of line orientation in the surround stimulus compared with the RF stimulus.

\footnotetext{
Received Oct. 2, 2000; revised Dec. 12, 2000; accepted Dec. 14, 2000.

We thank Peter De Weerd for invaluable help during the early stages of this project and Nurit Bloom for assistance in animal testing and data analysis.

Correspondence should be addressed to Dr. Andrew F. Rossi, Laboratory of Brain and Cognition, National Institute of Mental Health, National Institutes of Health, Building 49, Room 1B80, 49 Convent Drive, MSC 4415, Bethesda, MD 20892. E-mail: rossi@ln.nimh.nih.gov.

Dr. Desimone's address: Laboratory of Neuropsychology, National Institute of Mental Health, National Institutes of Health, Bethesda, MD 20892.

Copyright (C) 2001 Society for Neuroscience 0270-6474/01/211698-12\$15.00/0
}

In addition to suppression, enhancement of V1 responses by surround stimuli has also been observed under some conditions. In one such condition, line elements in the surround enhanced the response to a line element in the RF when they were collinear with it (Nelson and Frost, 1985; Kapadia et al., 1995; Polat et al., 1998). Similarly, excitatory effects of surround stimulation were observed in studies of figure-ground segregation using texture stimuli (Lamme, 1995; Zipser et al., 1996; Lee et al., 1998). In these experiments, the response to a texture "pop-out figure," formed by a texture boundary (difference in orientation of line elements) between the figure and a uniform background texture, was larger than the response to the uniform-texture background alone. However, there is some discrepancy among the different studies in the spatial scale over which response enhancement from the surround is observed. Whereas colinearity enhancement effects are limited to surround stimuli in the immediate vicinity of the RF (Nelson and Frost, 1985; Kapadia et al., 1995), V1 activity signaling pop-out of a figure defined by an orientation-defined boundary has been reported for boundaries located up to $4^{\circ}$ from the RF (Zipser et al., 1996). Enhancement effects over such large retinal extents suggest that V1 neurons play an important role in figure-ground segregation.

We therefore reinvestigated the role of contextual surround stimuli in modulating V1 responses over a wide range of distances. If figure-ground information is represented in V1, as has been proposed (Lamme, 1995; Zipser et al., 1996; Lee et al., 1998), then contextual modulation should exhibit some degree of invariance for figures of different sizes, provided the boundaries are beyond the RF. Alternatively, if contextual modulation is confined to surround stimuli in the immediate vicinity of the RF, then the contextual effects observed in V1 may represent an 
earlier stage of processing that contributes to the segregation of figures from their backgrounds in subsequent cortical areas.

\section{MATERIALS AND METHODS}

Surgery. Experiments were performed on two male Macaca mulatta, weighing 8-11 kg. Each monkey was surgically implanted with a head post, a scleral eye coil, and a recording chamber (one monkey had bilateral recording chambers). Surgery was conducted under aseptic conditions with isofluorane anesthesia (for details, see Miller et al., 1993). Antibiotics and analgesics were administered after the operation. The skull remained intact during the surgery. Subsequently, small holes ( $\sim 4 \mathrm{~mm}$ in diameter) were drilled within the recording chambers under ketamine anesthesia and xylazine analgesic. All experimental procedures were performed in accordance with National Institutes of Health guidelines and approved by the National Institute of Mental Health Intramural Animal Care and Use Committee.

Recording technique. Neuronal recordings were made through a surgically implanted chamber overlaying the operculum of area V1. Recordings were made from three hemispheres in two monkeys. Activity from single neurons or clusters of neurons was recorded extracellularly from single tungsten microelectrodes (Frederick Haer and Co., Brunswick, $\mathrm{ME}$ ), which were inserted through the intact dura by means of a hydraulic microdrive. For each penetration, the electrode was advanced through the dura mater at a very slow rate $(2 \mu \mathrm{m} / \mathrm{sec})$ to reduce the possibility of suppression artifacts resulting from the deformation of the cortical surface by the electrode. The RFs of the V1 neurons studied were at eccentricities in the range of $2-6^{\circ}$ in the lower contralateral visual field. To confirm that the recordings were in $\mathrm{V} 1$, the RF positions for neurons in each experiment were graphed to ensure that the location of microelectrode penetrations followed the orderly retinotopic mapping of the visual field onto V1. The approximate laminar position of the recording electrode was determined by the depth of the microelectrode and the characteristic features of layer 4 (namely, high spontaneous firing rate and brisk "on" and "off" responses). No effort was made to select neurons from a particular layer of cortex, although the majority of the recordings were made from layers 2 and 3. The only criterion for exclusion from recording was whether the signal was not clearly isolated or whether the RF of the neuron could not be mapped consistently. In some instances, two neurons could be recorded simultaneously and differentiated on the basis of the size and shape of the spike waveform. An on-line spike-sorting computer was used to classify these spikes by means of a template-matching procedure. The temporal resolution of the spike acquisition system was $1 \mathrm{msec}$.

Recordings from area V2 were made in one monkey by advancing the electrode through V1 into the posterior bank of the lunate sulcus. The V2 recordings were made primarily as a control to investigate the possibility that the surface recordings made in V1 were affected by deformation of the cortical surface associated with the advancing of the electrode through the dura mater. The RFs of the V2 neurons studied were at eccentricities in the range of $4-6^{\circ}$ in the lower contralateral visual field.

Stimuli. Stimuli were presented on a computer monitor, driven by a Number Nine Corporation graphics board with $640 \times 480$ pixel resolution and a frame rate of $60 \mathrm{~Hz}$. The screen was $26 \mathrm{~cm}$ wide and $21 \mathrm{~cm}$ high and was viewed from a distance of $57 \mathrm{~cm}$. The static texture stimuli were designed after those used by Zipser et al. (1996). The texture consisted of black bars on a gray background. The gray background was identical to the uniform gray screen presented during the intertrial period, which had a luminance of $18 \mathrm{~cd} / \mathrm{m}^{2}$. The texture element size and spacing were $0.7 \times 0.04^{\circ}$ and $0.3 \times 0.3^{\circ}$, respectively. The orientation of the texture elements was either 45 or $-45^{\circ}$ from vertical. These orientations were chosen to avoid luminance differences that can occur when presenting elements at other orientations. The orientation of the texture elements within the RF was chosen as the orientation (either 45 or $-45^{\circ}$ ) that elicited the better response in previous testing with $2^{\circ}$ texture patches that were centered in the RF. Figure $1 A$ shows the spatial configuration of the visual display relative to the fixation spot and the $\mathrm{RF}$. The boundaries of the figure, shown in Figure $1 A$ as a dashed circle, were centered on the RF.

Figure $1 B-E$ illustrates the four configurations of the texture stimulus that were used in these experiments. Neuronal responses were compared for a homogeneous texture field (Fig. $1 B$ ), a texture in which a figure was defined by a difference in the orientation of the elements compared with the background texture (Fig. $1 C$ ), a texture figure alone with no background (Fig. $1 D$ ), and a texture surround (annulus) alone (Fig. $1 E$ ). For each of the configurations in which a figure was present (Fig. $1 C-E)$, the
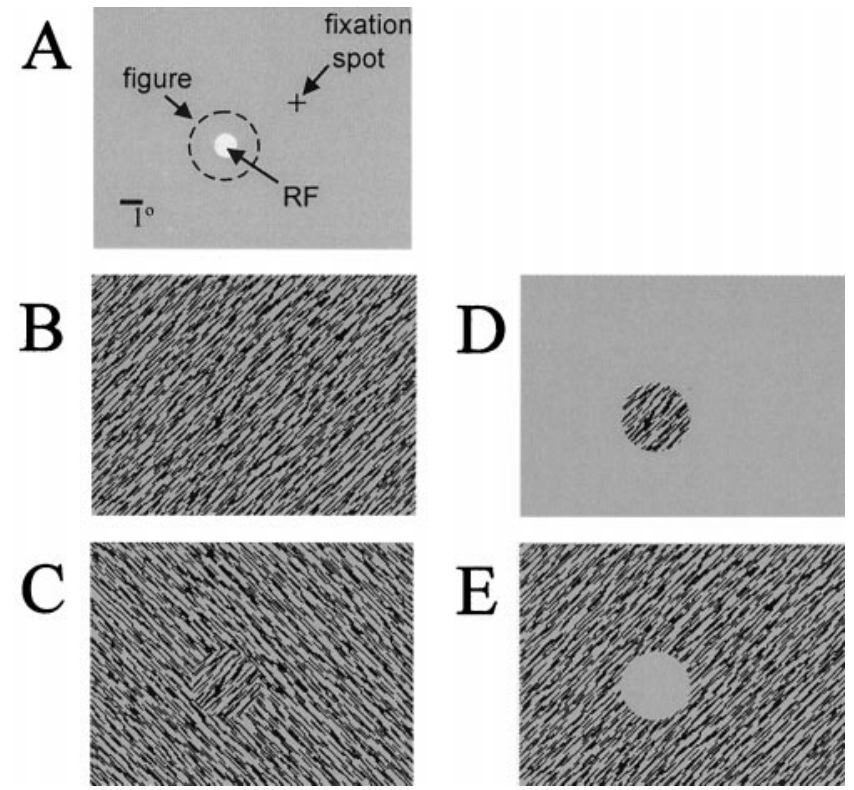

Figure 1. The four stimulus configurations used for studying contextual interactions. $A$, The spatial configuration of the visual display relative to the fixation spot and the RF (represented by the white disk). The figure, shown as a dashed circle, was centered on the RF. $B$, Uniform texture. $C$, Orientation-defined figure. $D$, Figure alone. $E$, Surround texture alone. For configurations shown in $B-D$, the pattern of texture elements within the figure was identical.

figure was centered on the RF. Note that although the configuration shown in Figure $1 E$ does not contain texture elements within the RF, a figure is nonetheless defined by the surround texture. Each neuron was tested with figure sizes from 1 to $5^{\circ}$ in diameter, and some neurons were tested with figures with diameters up to $12^{\circ}$. For each neuron, the luminance $(\mathrm{L})$ contrast of the texture elements was chosen as the contrast that elicited a response that was $\sim 80 \%$ of the maximum response to a texture stimulus (typically between 6 and $48 \%$ Michelson contrast $[(\mathrm{L} \max -\mathrm{L} \min ) /(\mathrm{L} \max +\mathrm{L} \min )])$, unless otherwise indicated in Results. The texture extended to the edges of the screen for those conditions in which a "background texture" was present (see Fig. $1 B, C, E$ ). Photodiodes were used to confirm that the luminance in the region of the RF did not change when the surround texture was presented alone (Fig. 1E). For every neuron tested, each stimulus condition was presented at least 20 times in a random sequence.

Behavioral testing. Recordings were made while the monkeys performed either a passive viewing task or a visual detection task. The temporal sequence of the passive viewing task was as follows. The monkey initiated a trial by holding a lever, a fixation spot appeared on the gray monitor screen, and the monkey foveated this spot. After the monkey maintained steady fixation for $300 \mathrm{msec}$, a texture stimulus appeared on the screen for $500 \mathrm{msec}$. The monkey received juice rewards for simply maintaining fixation throughout the trial. Recordings were made from both monkeys while they performed this passive viewing task.

To determine whether there was a task-related component to the neuronal response, one monkey was also trained on a visual detection task. For this task, the monkey was rewarded for detecting a texture figure in the display, using a Go-No-Go response paradigm. This monkey was rewarded for releasing a lever within $500 \mathrm{msec}$ (Go response) in trials in which a figure was present in the display and for holding the lever for a total of $2 \mathrm{sec}$ (No-Go response) in trials in which there was no figure present (uniform texture). The monkey performed this task for all stimulus configurations (Fig. $1 B-E$ ). There was an equal number of Go and No-Go trials, and the different stimulus configurations were randomly interleaved in a given session.

Fixation was monitored using the scleral search coil technique with a single coil in one eye. Vertical and horizontal eye position signals were stored at the temporal rate of $60 \mathrm{~Hz}$. The eye coil system was calibrated (for gain and offset) for each monkey at the beginning of each recording session. The peak-to-peak noise in the eye position signal did not exceed $0.1^{\circ}$ when a stable coil was placed in the magnetic field. Steady fixation 
required that the eye position of the monkey remain within a $1^{\circ}$ diameter fixation window (not visible in the stimulus display) that was centered on the fixation spot. Trials were aborted without reward if the gaze drifted $>0.5^{\circ}$ from the center of the fixation window.

Receptive field mapping. To estimate the size and location of the RF of a neuron, flashing bars (luminance increments and decrements) of variable size and orientation were presented in the region of the RF as the monkey fixated. The luminance contrast of the bars was $96 \%$ Michelson contrast, and the background luminance of the screen was $18 \mathrm{~cd} / \mathrm{m}^{2}$. By the use of these initial estimates, quantitative mapping of the RF was accomplished by presenting rectangular patches of texture (the same as that described above) in a $3 \times 3$ grid that was centered on the RF. The size of the texture patch, or element of the grid, ranged from 0.3 to $2.4^{\circ}$ (with a resolution of $0.1^{\circ}$ ) so that response maps of various spatial resolutions were generated for each neuron. The extent of the RF was determined to be the size of the texture patch that elicited a response at the center grid position and not in the surrounding positions. The orientation of the texture elements (either 45 or $-45^{\circ}$ ) was chosen to be the orientation closer to the preferred orientation of the neurons. The luminance contrast of the texture elements was $96 \%$. We found that the $\mathrm{RF}$ areas determined using this mapping method were typically $10 \%$ larger than those determined by qualitative mapping. RFs in V1 (between 2 and $6^{\circ}$ eccentricity) were typically between 0.5 and $1.5^{\circ}$ in diameter. RFs in V2 (eccentricities between 4 and $6^{\circ}$ ) were found to be between 0.5 and $4^{\circ}$ in diameter. Coarse orientation selectivity and contrast sensitivity were measured at each recording site using a texture stimulus $\left(2 \times 2^{\circ}\right)$ centered on the RF. No attempt was made to classify the neurons as "simple" or "complex." To ensure that the RF had not changed over the testing period, the RF mapping procedure was repeated after testing with the texture stimuli was completed for each neuron.

Data collection and analysis. Behavioral and physiological data were collected using Cortex acquisition software (http://cog.nimh.nih.gov/cortex) and were analyzed with MATLAB (The MathWorks, Inc., Natick, MA). For a given stimulus condition, responses to 20-50 stimulus presentations were collected. These were compiled into an average response histogram with a bin width of $5 \mathrm{msec}$, which was then used to compute the modulation index and the response onset latency. The average response was defined as the mean firing rate of the neuron for a period of $200 \mathrm{msec}, 50 \mathrm{msec}$ after the onset of the neuronal response. This response window was chosen to capture the average firing rate, excluding the initial onset transient. The modulation index for a given stimulus condition was defined as the average response elicited by the figure stimulus (see Fig. $1 C-E$ ) divided by the average response to the uniform texture (Fig. 1B). This index was used to facilitate comparison of our results with previous reports of contextual modulation in V1 (Lamme, 1995; see Zipser et al., 1996).

The response onset latency was calculated, first, by fitting a Poisson function to the distribution of the firing rate of the neuron for the period of $100 \mathrm{msec}$ before the onset of the stimulus. The response latency was taken to be the time corresponding to the first bin of the average response histogram after stimulus onset that (1) had a firing rate that exceeded a probability of $p=0.01$ for the baseline distribution, (2) was immediately followed by a bin that exceeded a probability of $p=0.01$ for the baseline distribution, (3) had an average response for $50 \mathrm{msec}$ after the first bin that was significantly different from the spontaneous firing rate ( $t$ test, $p<0.05$, one tail), and (4) occurred within $200 \mathrm{msec}$ of the stimulus onset. This method was derived, in part, from that used by Maunsell and Gibson (1992). If these statistical criteria were not satisfied, the response latency was not included in the population analysis.

To determine whether the average response to a given stimulus was significantly different from the response to the uniform texture, a $t$ test $(p<0.05$, one tail) was performed on the trial-by-trial firing rates. To avoid the assumption of homogeneity of variance in the neuronal firing rate, the calculation of the Student's $t$ test for unequal variance was used. As with the averaged spike histograms, the average response for a given trial was defined as the mean firing rate of the neuron for a period of $200 \mathrm{msec}, 50 \mathrm{msec}$ after the onset of the neuronal response.

\section{RESULTS}

We recorded from 135 sites in area V1 and 32 sites in area V2. Of these recording sites, we analyzed in detail 130 (V1, 73 single-unit and 29 multiunit; V2, 24 single-unit and 4 multiunit) that gave significant responses to the texture stimuli within the RF, were well isolated, and were held long enough to collect data for at least 20 presentations of each stimulus condition. There were no quantitative differences in the responses of the single-unit and multiunit recordings under these stimulus conditions, and they have therefore been combined in all of the subsequent analyses. All of the V1 recording sites were located on the operculum and had RF eccentricities between 2 and $6^{\circ}$, and all of the V2 sites were located in the posterior bank of the lunate sulcus and had RF eccentricities between 4 and $6^{\circ}$.

\section{Stimulation with orientation-defined figures: V1 recordings}

This experiment was designed to examine how the response to texture within the RF is influenced by the visual context in which the texture is presented. The context was manipulated by changing the orientation of the texture elements in the surround region of the RF relative to the orientation of elements in a disk-shaped figure centered on the RF (see Fig. 1C). The texture of the disk-shaped figure was always identical to the corresponding region of the uniform-texture condition (see Fig. $1 B, C$ ).

The responses to orientation-defined figures at various sizes (Fig. $1 C$ ) were compared with the response to the uniform texture (Fig. $1 B$ ). Figure 2 shows the responses of three example neurons to orientation-defined figures with sizes of 1,2 , and $4^{\circ}$ in diameter. The gray line in each graph represents the average response of the neuron to the uniform texture, whereas the black line represents the response to the orientation-defined figure. For the neuron in Figure 2, example $A$, the response to the $1^{\circ}$ orientationdefined figure was significantly larger than was the response to the uniform texture ( $p<0.05$, one-tailed $t$ test). Because the RF of this neuron $\left(1.2 \times 1.0^{\circ}\right)$ was larger than the $1^{\circ}$ orientation-defined figure, the enhanced response to the figure compared with the uniform texture was likely caused by the boundary contour of the figure falling within the RF. For conditions in which the orientation-defined figure was 2 or $4^{\circ}$, that is, large enough for its boundary to be outside the RF, there was no difference between the average responses to the orientation-defined figure and the uniform texture (Fig. 2, example A, lower two graphs). Figure 2, example $B$, illustrates a similar effect, namely, that the average response to the $1^{\circ}$ figure, but not the 2 or $4^{\circ}$ figure, was significantly larger than was the response to the uniform texture ( $p<0.05$, one-tailed $t$ test). For this neuron, the response to the orientation-defined figure was dramatically reduced when its boundary was located $>0.5^{\circ}$ from the putative RF border $\left(0.7 \times 0.7^{\circ}\right)$.

Approximately $55 \%$ of our sample of V1 neurons showed similar effects, namely, that the magnitude of the response to an orientation-defined figure was dependent on the distance between the boundary of the figure and the border of the RF. The remaining $45 \%$ of neurons did not show any difference in the response to the orientation-defined figure and the uniform texture, regardless of the size of the figure. For these neurons, all that mattered was that texture was within the RF. An example of such a neuron is shown in Figure 2, example $C$.

A modulation index was used to quantify the difference between the responses to the orientation-defined figure and the uniform texture. This index was the ratio of the average response elicited by the figure to that elicited by the uniform texture. An index $>1.0$ indicates that the average response elicited by the orientation-defined figure was greater than that elicited by the uniform texture.

Figure 3 illustrates the distribution of the modulation index for neurons that were studied with orientation-defined figures $1-5^{\circ}$ in 

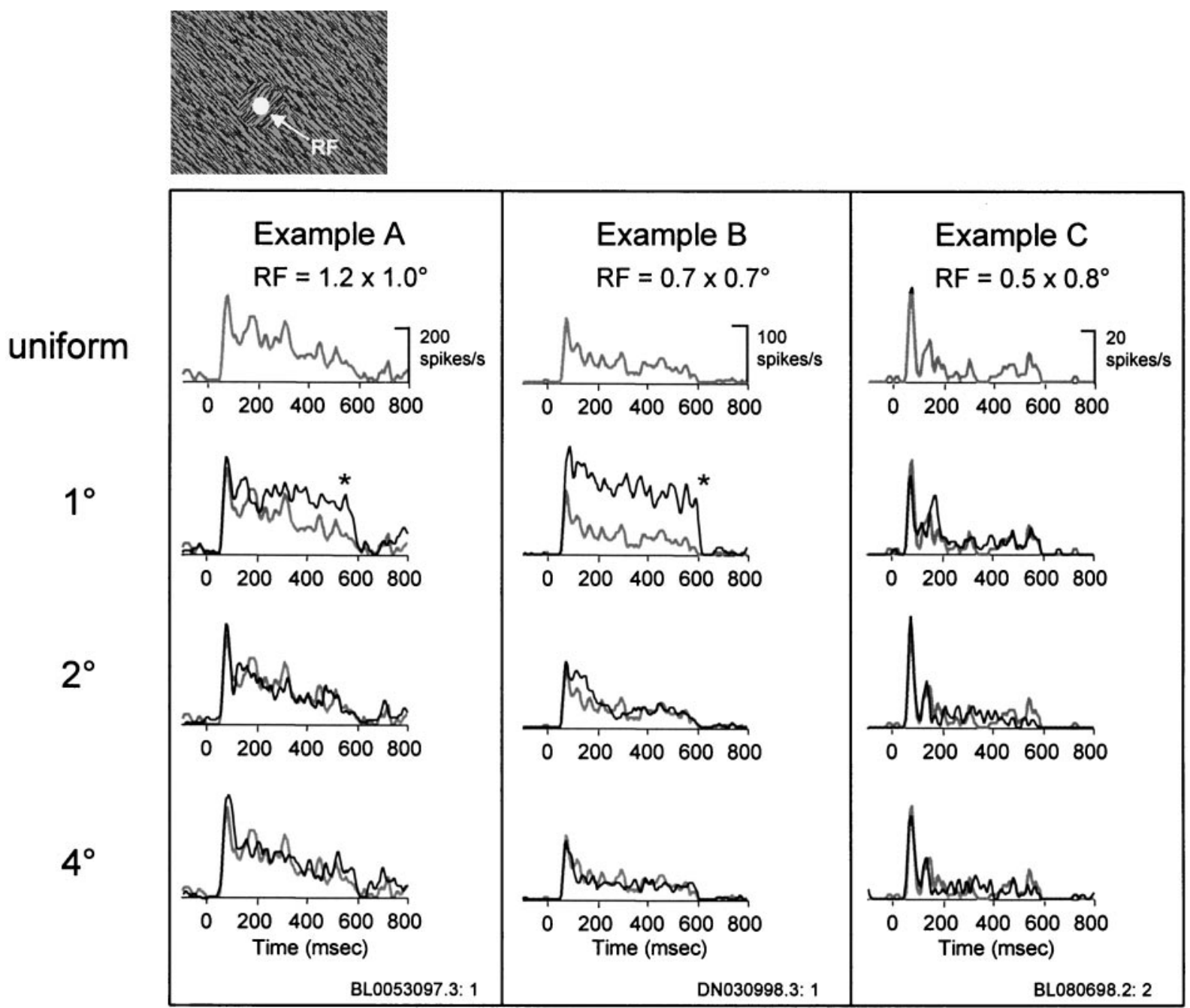

Figure 2. Response modulation with orientation-defined figures. Examples $(A-C)$ of the responses of three isolated V1 neurons to the uniform texture and orientation-defined figures of 1,2 , and $4^{\circ}$ in diameter. The upper left panel illustrates the figure/ground stimulus relative to the RF (represented by the white disk). The figure was always aligned on the center of the RF. RF sizes of the three examples are indicated within each panel. The black lines in examples $A-C$ correspond to the average response of the neuron to the orientation-defined figure, the diameter of which is indicated in the leftmost column. For comparison, the gray lines in examples $A-C$ correspond to the average response to the uniform texture. An asterisk indicates that the response to the orientation-defined figure was significantly greater than the response to the uniform texture (1-tailed $t$ test, $p<0.05$ ).

diameter. Neurons with RFs $>1^{\circ}(n=5)$ were excluded from this sample so that we could control, across the population of neurons, the range of distances between the boundaries of the figure and the RF border. The black and gray arrows at the top of each histogram indicate the mean and the median of the distributions, respectively. The mean of the distribution was significantly $>1.0$ for those stimulus conditions in which the orientation-defined figure was 1 or $2^{\circ}$ in diameter (one-tailed $t$ test, $p<0.05$ ). For figure sizes $>2^{\circ}$, the modulation index was approximately normally distributed around a value of 1.0. These data thus indicate that, across the population of neurons with RFs $1^{\circ}$ or less, the contextual modulation was significant only for the stimulus conditions in which the boundary of the orientation-defined figure was within $1.5^{\circ}$ of the RF center. Thus, there was no evidence that V1 neurons responded preferentially to "figures" over a large range of sizes.

Because the spatial extent of the contextual modulation was unexpectedly small compared with findings in some previous reports, we tested whether the spatial extent might increase if the monkey actively used information from the stimulus in the per- formance of a task. Accordingly, one animal was trained to detect the presence of the orientation-defined figure in a Go-No-Go paradigm, using an orientation-defined figure with a diameter of $3^{\circ}$. This size was chosen to be beyond the range of significant contextual modulation effects observed in the fixation task described above. This monkey was rewarded for releasing a lever within $500 \mathrm{msec}$ (Go response) in trials in which a figure was present in the display and for holding the lever for a total of $2 \mathrm{sec}$ (No-Go response) in trials in which no figure was present (uniform texture). The performance of the monkey averaged 94\% correct for this task. Figure $4 A$ compares the average modulation index for neurons recorded during this figure detection task $(n=$ 31) with those recorded during experiments in which the monkey was rewarded for simply maintaining fixation $(n=97)$. Contrary to expectations, the detection task did not increase the magnitude of contextual modulation ( $t$ test, $p=0.84$ ).

Another possibility was that the spatial extent of contextual modulation would be greater at lower stimulus contrast. In the experiments thus far, the luminance contrast of the texture elements was chosen for each neuron as the contrast value that 


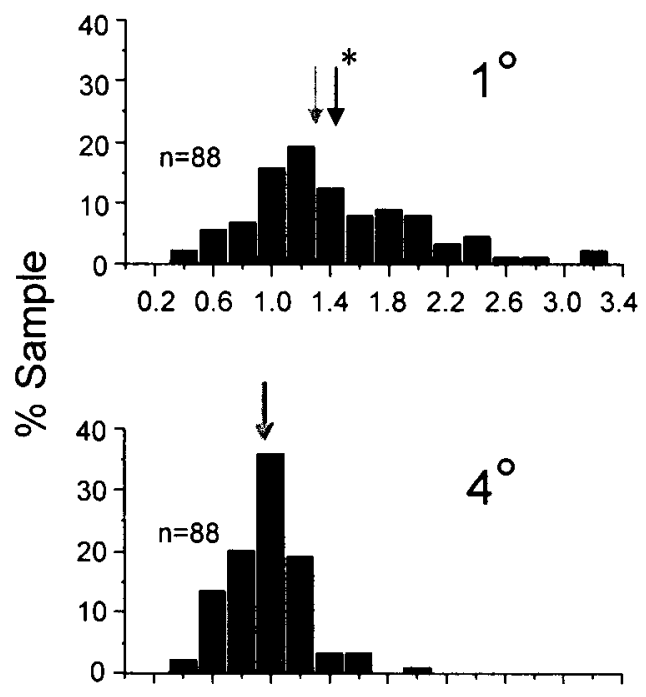

$\begin{array}{llllllllll}0.2 & 0.6 & 1.0 & 1.4 & 1.8 & 2.2 & 2.6 & 3.0 & 3.4\end{array}$

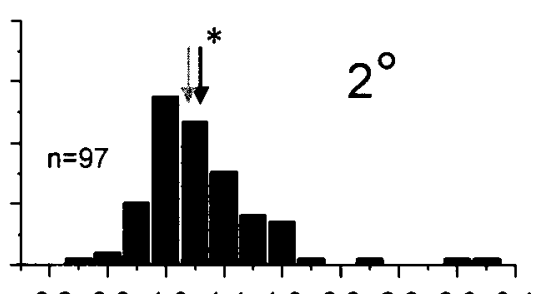

$\begin{array}{llllllllll}0.2 & 0.6 & 1.0 & 1.4 & 1.8 & 2.2 & 2.6 & 3.0 & 3.4\end{array}$

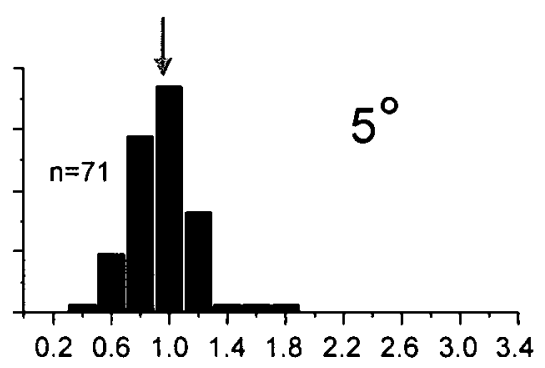

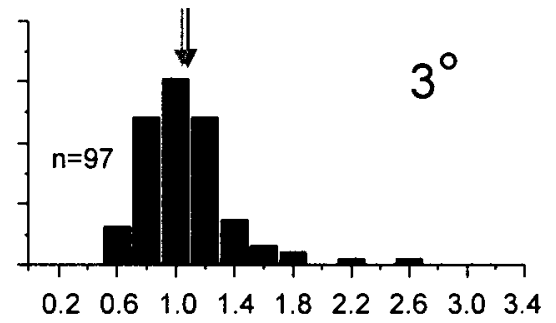

$\begin{array}{lllllllllll}0.2 & 0.6 & 1.0 & 1.4 & 1.8 & 2.2 & 2.6 & 3.0 & 3.4\end{array}$

\section{Modulation Index}

Figure 3. Distribution of the modulation index for figure/ground stimulation. Histograms indicate the distribution of the modulation index for orientation-defined figures ranging from 1 to $5^{\circ}$ in diameter. The positions of the gray and black arrows at the top of each histogram correspond to the median and mean of the population, respectively. Data shown are for single and multiunit sites with RF sizes $1^{\circ}$ in diameter or smaller (see Table 1 ). A modulation index of 1.0 indicates that the response to the orientation-defined figure was equivalent to the response to the uniform texture. The asterisk indicates that the mean of the distribution was significantly $>1.0$ (1-tailed $t$ test, $p<0.05$ ).

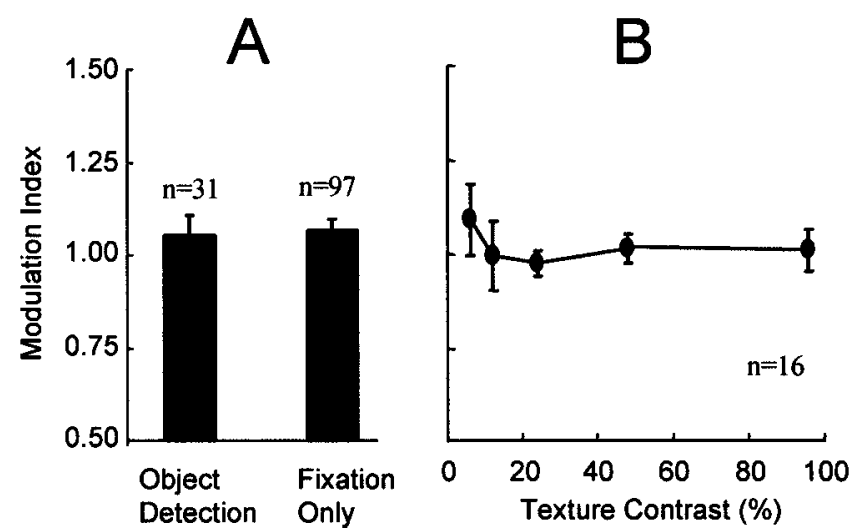

Figure 4. The effect of behavior and texture contrast on response modulation. The average modulation index was calculated for orientationdefined figures of $3^{\circ}$ in diameter. $A$, Comparison of the modulation index for identical stimulus conditions in which the monkey was rewarded either for detecting the presence of a figure in the display (average index $=1.05$ ) or for simply maintaining fixation during the trial (average index $=1.07$ ). $B$, Average modulation index for $16 \mathrm{~V} 1$ neurons that were studied with texture contrasts of $6,12,24,48$, and $96 \%$. The modulation index was calculated for the responses to a $3^{\circ}$ orientation-defined figure while the monkey performed the figure detection task. Error bars are SEs.

elicited a response that was $\sim 80 \%$ of the maximum response to a texture stimulus (typically between 6 and $48 \%$ Michelson contrast). It was conceivable, however, that the degree of contextual modulation would vary with response strength and, although we were careful to avoid saturating the response of the neurons, we might observe greater contextual modulation with lower contrast textures. To test this, we examined the responses of 16 neurons under conditions in which the texture stimuli were presented at luminance contrasts ranging from 6 to $96 \%$. Again, the diameter of the orientation-defined figure was $3^{\circ}$. Figure $4 B$ illustrates that the average modulation index was not significantly affected by changes in the contrast of the texture elements. Although there was an elevation of the average modulation index at the lowest contrasts, the results of a one-way ANOVA indicate that there was no difference between the average values of the modulation index across the texture contrasts $(F=0.484 ; p=0.747)$.

In summary, the enhanced response to the orientation-defined figure was present only for conditions in which the defining border of the figure was in close proximity to the RF border. In addition, we found that the magnitude of contextual modulation was not significantly affected by changes in either the nature of the behavioral task or the contrast of the texture elements.

\section{Boundary effects, surround suppression, and surround excitation: V1 recordings}

In the experiments described thus far, contextual modulation was observed only under conditions in which figure diameters were $2^{\circ}$ or less (typical boundaries within $0.5^{\circ}$ of the RF border), suggesting that the neurons were responding to the presence of the orientation-defined boundary within their RFs. This is not an unlikely possibility because the precise RF borders are difficult to determine in awake monkeys. Others have shown that, for texture stimuli, V1 neurons respond vigorously to the presence of an orientation-defined contour placed within their RFs, independent of the orientation of the elements that comprise the texture (Gallant et al., 1994; Lee et al., 1998). To explore the role of orientation-defined texture contours further, we examined the degree of response modulation to figures of varying sizes, under conditions in which we manipulated the cues that define the boundary of the figure.

Figure 5 illustrates the responses of a V1 neuron to three texture figures on different backgrounds (Fig. $5 B-D$; black lines in graphs) compared with the response elicited by the uniform texture alone (Fig. 5A-D; gray lines in graphs) for figures sizes of 1 , 2 , and $4^{\circ}$. For this neuron, there was an enhanced response to the 1 and $2^{\circ}$ orientation-defined figures, but not to the $4^{\circ}$ figure, when 


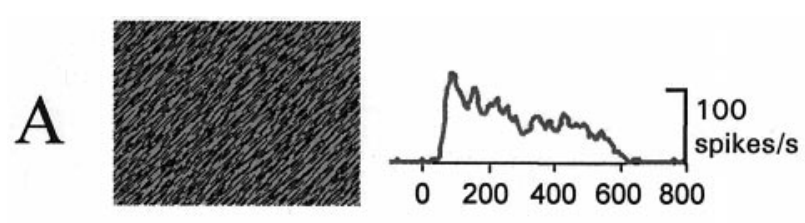

Figure Diameter
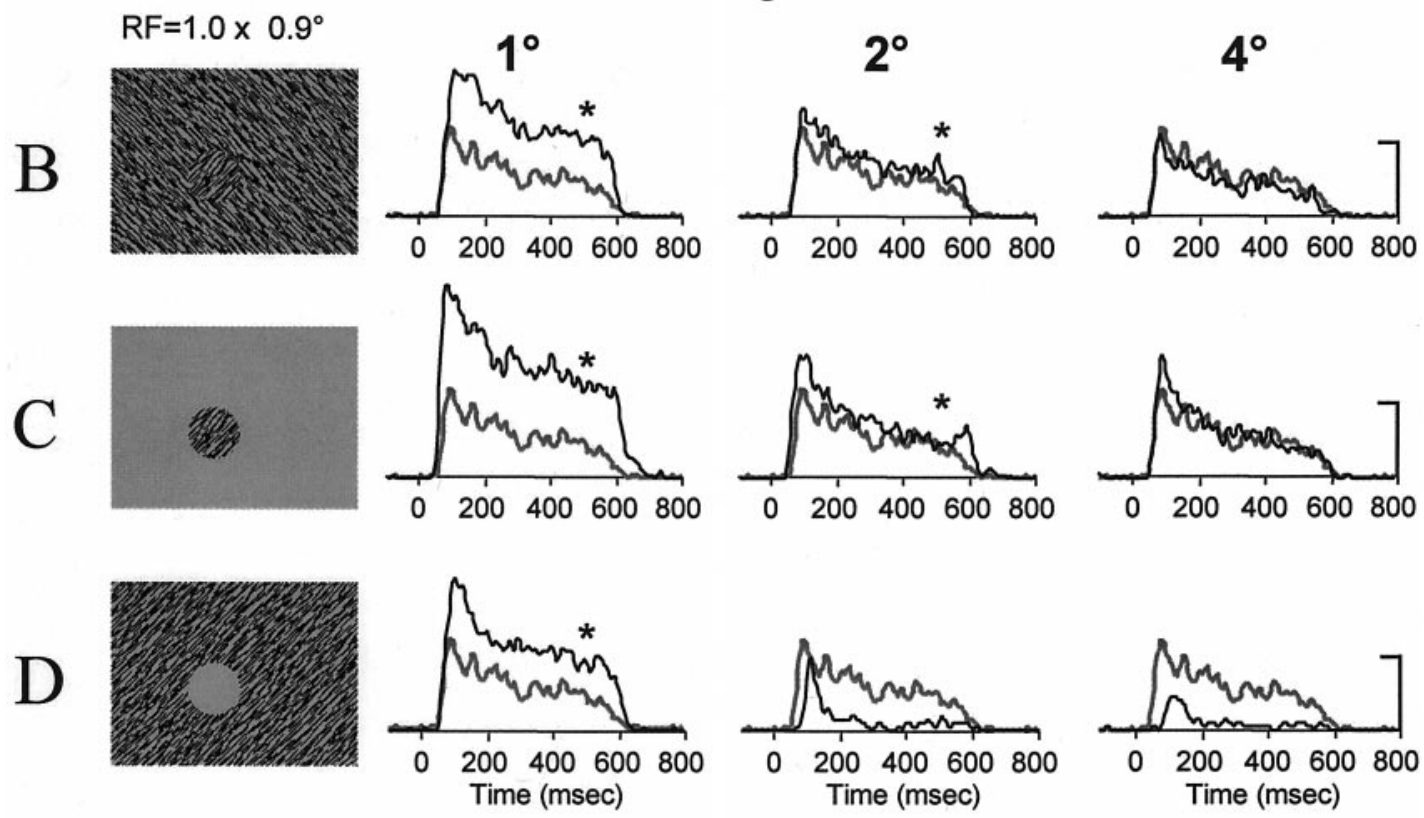

DN022498.3:1

Figure 5. Effects of stimulus configuration and figure size on response modulation. $B-D$, An example of the response of a single V1 neuron to different sizes of the orientation-defined figure $(B)$, figure alone $(C)$, and the surround-texture condition $(D)$. A, The response to the uniform texture. The black line in $B-D$ corresponds to the average response of the neuron to the stimulus configuration depicted in the leftmost column. For comparison, the gray line in $A-D$ corresponds to the average response to the uniform texture. An asterisk indicates that the response to the figure stimulus was significantly greater than the response to the uniform texture (1-tailed $t$ test, $p<0.05$ ). The size of the $\mathrm{RF}$ was $1.0 \times 0.9^{\circ}$. The luminance contrast of the texture elements was $24 \%$.

compared with the response elicited by the uniform texture (Fig. $5 A$ ). The same enhanced response for small texture figures (compared with the uniform-texture background) was observed for textured figures on a gray background, i.e., without a texture surround (Fig. 5C). Thus, for texture figures on both gray and textured backgrounds, the response was enhanced for those stimuli in which the diameter of the figure was approximately the same size or smaller than the diameter of the RF.

One interpretation of this finding is that it is the presence of the texture boundary within or close to the RF border that causes the enhanced response relative to that of the uniform-texture background. As the texture boundary is moved farther from the RF border, the enhancement is eliminated. A second, alternative, interpretation is that the only excitatory response derives from the texture elements contained within the RF, and this response is partially suppressed when the texture extends beyond the border of the RF into the surround. That is, according to this interpretation, the RF has a suppressive surround, and the response to the texture inside the RF is increasingly suppressed as the texture spreads beyond the RF boundary into the surround. The manipulation shown in Figure $5 D$, described below, provides evidence favoring the first interpretation.

Figure $5 D$ shows a stimulus configuration in which the surround texture was presented in isolation, as a texture annulus. The largest dimension of the RF of this neuron was $1^{\circ}$, and it exhibited the typical enhancement to orientation-defined figures when the figure boundary was within or close to the RF border. The critical condition was when the neuron was stimulated with the surroundtexture annulus. In this configuration, the neuron gave a significant response for annuli with inner diameters of 1,2 , and $4^{\circ}(t$ test, $p<0.01$; relative to the response to the spontaneous firing rate). That is, with a texture stimulus confined entirely to the surround, the presence of a texture boundary near the RF border elicited an excitatory response. This suggests that texture stimuli in the surround are not necessarily suppressive and, by extension, that the location of the texture boundary within or near the RF was the most likely cause of the enhancement observed in the response to the orientation-defined figure described in the previous section. The excitatory effect of surround stimuli will be considered further in a later section.

Figure 6 shows the results of the same stimulus manipulations as in Figure 5, but the response histograms are based on the average responses of $88 \mathrm{~V} 1$ neurons with RFs $1^{\circ}$ in diameter or smaller. As in the single neuron example in Figure 5, the averaged responses across neurons demonstrate the relationship between the degree of response modulation and the distance of the figure 


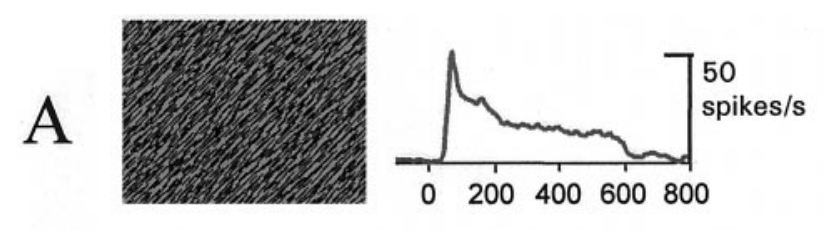

\section{Figure Diameter}
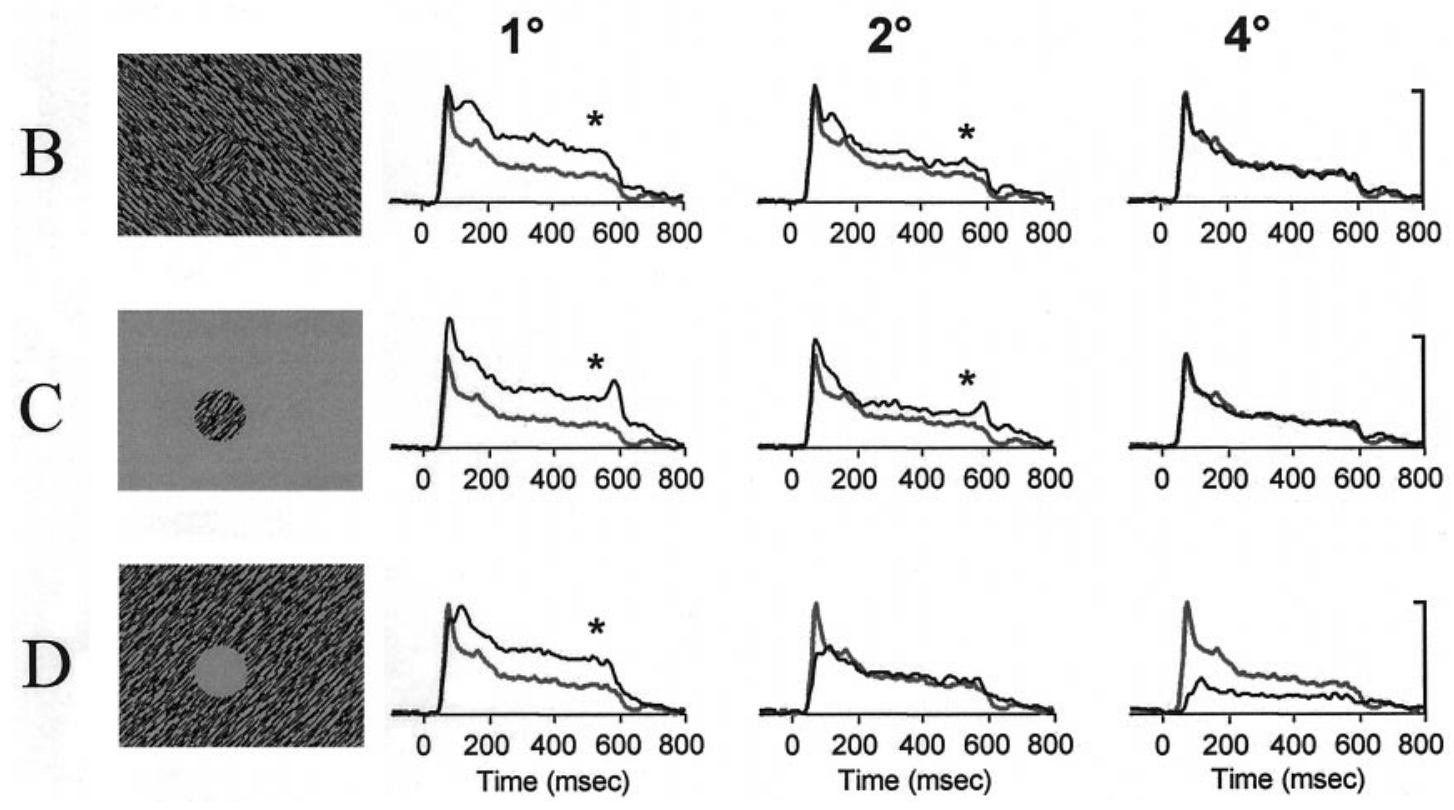

Figure 6. Average effects of stimulus configuration and figure size on response modulation. The format is identical to that in Figure 5. B-D, The average responses of $88 \mathrm{~V} 1$ neurons to different sizes of the orientation-defined figure $(B)$, figure alone $(C)$, and the surround-texture condition $(D)$. $A$, The average response to the uniform texture. The black line in $B-D$ corresponds to the average response of the population of neurons to the stimulus configuration depicted in the leftmost column. For comparison, the gray line in $A-D$ corresponds to the average population response to the uniform texture. An asterisk indicates that the average response to the figure stimulus was significantly greater than the average response to the uniform texture (1-tailed $t$ test, $p<0.05$ ).

boundary from the RF border. On average, there were significantly larger responses to both the 1 and $2^{\circ}$ orientation-defined figures, but not to the $4^{\circ}$ figure, when compared separately with the response elicited by the uniform texture (one-tailed $t$ test, $p<$ $0.05)$. The same decrease in response modulation with figure size was observed for textured figures without a texture surround (Fig. $6 C$ ) and for the surround texture alone (Fig. 6D). Figure 6, $1^{\circ}$ column, illustrates that, regardless of the figure configuration, there was approximately the same relative increase in the response to a $1^{\circ}$ figure when compared with the response to the uniform texture. The response to the $1^{\circ}$ figure defined by the surround texture alone (Fig. $6 \mathrm{D}, 1^{\circ}$ panel) demonstrates that, on average, the neurons responded strongly to the presence of the stimulus contour, despite the absence of texture elements within the RF center. In addition, the response elicited by the surround texture alone (Fig. 6D) was, on average, significantly greater than was the average spontaneous rate for annuli with inner diameters of 1,2 , and $4^{\circ}$ ( $t$ test, $\left.p<0.01\right)$.

To evaluate quantitatively the population response to the different stimulus conditions, we computed the average modulation index as a function of figure diameter for the sample of V1 neurons with RFs $1^{\circ}$ in diameter or smaller. As illustrated in Figure $7 A$, the open circles and filled squares show that the average modulation index was $>1.0$ for orientation-defined figures and figures alone, respectively, with diameters $<4^{\circ}$. For the surround texture alone (Fig. $7 A$, filled triangles), the modulation index was
$>1.0$ for surrounds with inner diameters $<3^{\circ}$. For surround textures with inner diameters $>2^{\circ}$, the modulation index was $<1.0$, indicating that the response was less than the response elicited by the uniform texture. However, even for surround textures with inner diameters as large as $5^{\circ}$, the average response of our sample of neurons was greater than the spontaneous activity. Therefore, there was an excitatory response to the inner border of the surround texture alone at distances from the RF for which response modulation was no longer observed for the orientation-defined figure or the texture figure alone.

To assess the significance of the average modulation indices for our population, we determined the proportion of neurons that showed a significantly greater response $(p<0.05$, one-tailed $t$ test) to a stimulus that contained a figure compared with the response to the uniform texture. Figure $7 B$ shows that, for each of the three stimulus configurations, the percentage of neurons that responded significantly better to a figure decreased proportionately as the diameter of the figure was increased. Regardless of the configuration of the stimulus, the percentage of our sample that showed a significant response to figure sizes of 4 and $5^{\circ}$ was at, or close to, the percentage that would be expected to occur by chance $(p=0.05$, indicated by the arrow in Fig. $7 B)$. For figure diameters $>1^{\circ}$, there were similar percentages of neurons demonstrating significant modulation elicited by the three different stimulus configurations. For figure diameters of $1^{\circ},>60$ and $>55 \%$ of our sample responded significantly better either to a 
Figure 7. Effects of boundary distance on response modulation. $A$, Average modulation index for each stimulus configuration plotted as a function of the figure diameter. The vertical position of the arrow represents the average modulation index calculated for the spontaneous firing rate (spontaneous rate/rate evoked by uniform figure). Error bars correspond to the SEMs. Open circles correspond to the orientation-defined figure, filled squares correspond to the figure alone, and filled triangles correspond to the surround texture alone. Data shown are for single and multiunit sites in $\mathrm{V} 1$ with RFs $1^{\circ}$ in diameter or less (see Table 1). B, Percentage of recording sites that exhibited a significantly higher firing rate in response to the figure stimulus as compared with the response to the uniform texture (1-tailed $t$ test, $p<0.05$ ) plotted as a function of figure diameter. The vertical position of the arrow represents the percentage of neurons that would be expected by chance. deg, Degree.

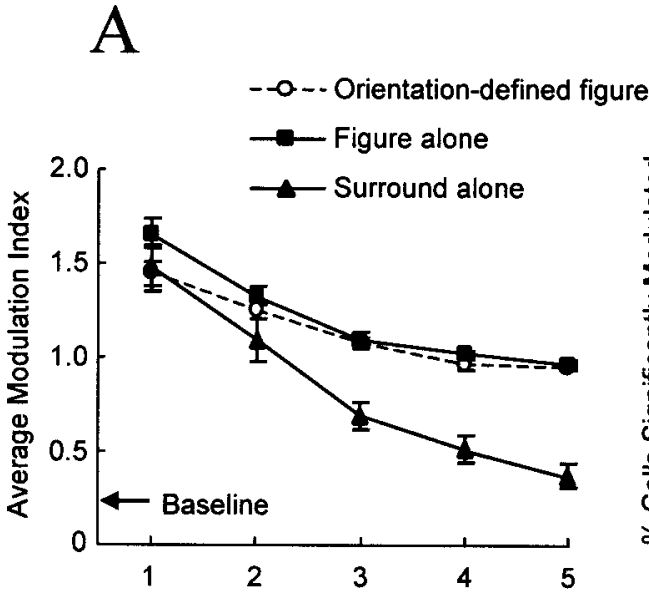

B

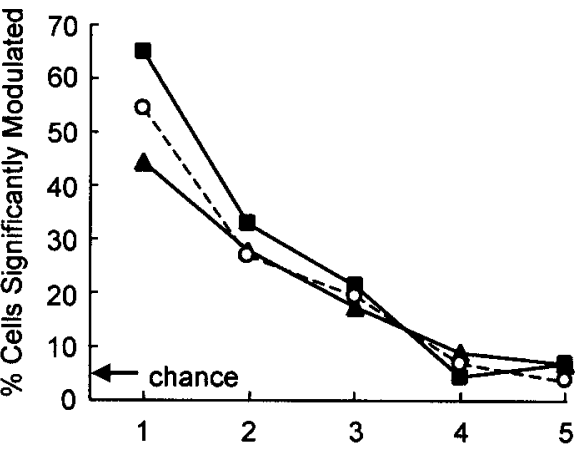

Figure Diameter (deg)

Table 1. Comparison of the incidence of response modulation observed with different texture configurations for V1 neurons with RF sizes $1^{\circ}$ or smaller

\begin{tabular}{llllll}
$\begin{array}{l}\text { Figure } \\
\text { diameter }\end{array}$ & $\begin{array}{l}\text { Number of } \\
\text { neurons }\end{array}$ & $\begin{array}{l}\text { A } \\
\text { Orientation- } \\
\text { defined figure }\end{array}$ & B & $\begin{array}{l}\text { C } \\
\text { Surround } \\
\text { alone }\end{array}$ & $\begin{array}{l}\text { D } \\
\text { Contextual modulation and } \\
\text { response to surround (A, C) }\end{array}$ \\
\hline $1^{\circ}$ & 88 & $48(54.5 \%)$ & $57(64.8 \%)$ & $82(93.2 \%)$ & $48(54.5 \%)$ \\
$2^{\circ}$ & 97 & $26(26.8 \%)$ & $32(33 \%)$ & $81(83.5 \%)$ & $22(22.7 \%)$ \\
$3^{\circ}$ & 97 & $19(19.6 \%)$ & $21(21.6 \%)$ & $54(55.6 \%)$ & $15(15.5 \%)$ \\
$4^{\circ}$ & 88 & $6(6.8 \%)$ & $3(3.4 \%)$ & $38(43.2 \%)$ & $3(3.4 \%)$ \\
$5^{\circ}$ & 71 & $3(4.2 \%)$ & $4(5.6 \%)$ & $32(45.1 \%)$ & $2(2.8 \%)$ \\
$8^{\circ}$ & 23 & 0 & 0 & $8(34.8 \%)$ & 0 \\
$12^{\circ}$ & 23 & 0 & 0 & $7(30.4 \%)$ & 0
\end{tabular}

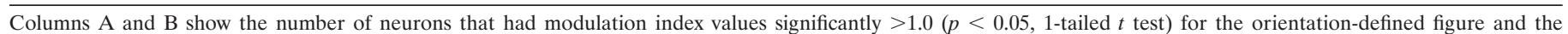

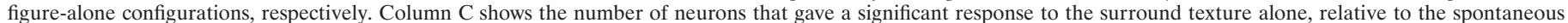

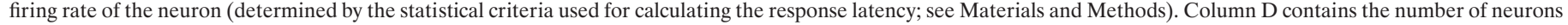
that exhibited both contextual modulation to the orientation-defined figure and a significant response to the surround texture when presented alone.

figure alone or to orientation-defined figures, respectively, compared with the response to the uniform texture. By contrast, $<45 \%$ of our sample showed a significantly greater response to the surround alone as compared with the uniform texture.

It is important to note in Figure 7 that, unlike the orientationdefined figure and figure-alone configurations, the response to the surround alone configuration for figure sizes $>1^{\circ}$ was not a modulation of the response to texture within the RF. Rather, it was a direct response to the surround stimulation alone. For $>93 \%$ of these neurons, there was a significant response to the surround alone as compared with their spontaneous firing rate. As shown in Table 1, columns A-D, for each of the figure diameters tested, ranging from 1 to $12^{\circ}$, fewer than five neurons in our sample exhibited a significant degree of contextual modulation to the orientation-defined figure and did not respond to the surround texture alone.

\section{V2 recordings}

The motivation for recording in V2 was to investigate the possibility that the surface recordings made in V1 were affected by deformation of the cortical surface associated with advancing the electrode through the dura mater. Specifically, it has been suggested by Zipser et al. (1996) that the expression of extra-RF contextual modulation is adversely affected by a general suppression of activity resulting from the advancing electrode. Although we did not suspect any suppression of activity in our recordings
Table 2. Changes in the modulation index with figure size for $28 \mathrm{~V} 2$ neurons tested with orientation-defined figures

\begin{tabular}{|c|c|c|c|c|c|}
\hline & \multicolumn{5}{|c|}{ Figure diameter } \\
\hline & $1^{\circ}$ & $2^{\circ}$ & $3^{\circ}$ & $4^{\circ}$ & $5^{\circ}$ \\
\hline Mean modulation index & $1.71 *$ & $1.21^{*}$ & 1.07 & 0.98 & 1.01 \\
\hline Median modulation index & 1.58 & 1.16 & 1.04 & 0.97 & 0.99 \\
\hline \multicolumn{6}{|c|}{ Number of neurons significantly } \\
\hline modulated & 13 & 6 & 3 & 1 & 1 \\
\hline
\end{tabular}

from area $\mathrm{V} 1$, it is conceivable that the contextual responses could have been preferentially affected by the compression of fibers in layer 1 . By recording in area V2 by means of a penetration through V1, we avoided this type of potential artifact.

Table 2 contains the mean and median values of the modulation index for $28 \mathrm{~V} 2$ neurons that responded to the orientationdefined figures. The response modulation observed in V2 was similar to that observed in V1 in several ways. First, the mean modulation index was significantly $>1.0$ for orientation-defined figures having a diameter of 1 or $2^{\circ}$, but not for figures with diameters $>2^{\circ}$ (one-tailed $t$ test, $p<0.05$ ). This indicates that the response modulation was greatest for those conditions in which the boundary of the figure was within, or in close proximity $\left(<1^{\circ}\right)$ 
Figure 8. The response onset latency increases as a function of the distance of surround stimulus from the RF center. $A$, Spatial configuration of the surround texture relative to the RF (represented by the white disk). The circular figure defined by the texture was centered on the RF. The luminance of the circular figure was identical to that of the background, so that the luminance of the figure remained constant during the presentation of the surround texture and the intertrial interval. $B$, Response of a single V1 neuron to the uniform texture and to surround textures presented $0.5-2.0^{\circ}$ from the center of the RF. The response to each stimulus is shown both as rasters (top of each pan$e l$ ), in which each dot is a single action potential and successive lines are different trials, and as average spike density functions (bottom of each panel). The stimulus duration was $500 \mathrm{msec}$ beginning at time 0 , indicated by the vertical line extending through each panel. For each of the surround stimulation conditions shown, the response to the texture surround was significantly greater than the spontaneous firing rate of the neuron (1-tailed $t$ test, $p<0.01)$. For this neuron, no significant response could be elicited to surround textures presented $>3^{\circ}$ from the center of the RF. $C$, The response latency of the same neuron plotted as a function of distance of the texture from the RF center. An asterisk indicates that the latency of the response to the surround stimulus was significantly greater than the latency of the response to the uniform texture (1tailed $t$ test, $p<0.01)$. The size of the RF, as measured with small bar stimuli, was $1.0 \times 1.0^{\circ}$. The luminance contrast of the texture elements was $48 \%$.
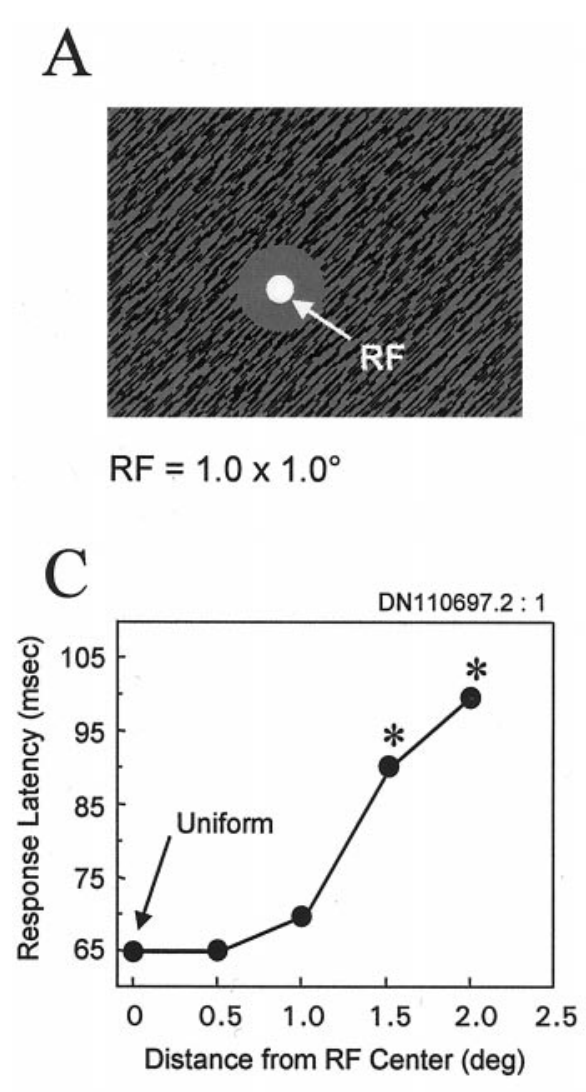

\section{B}

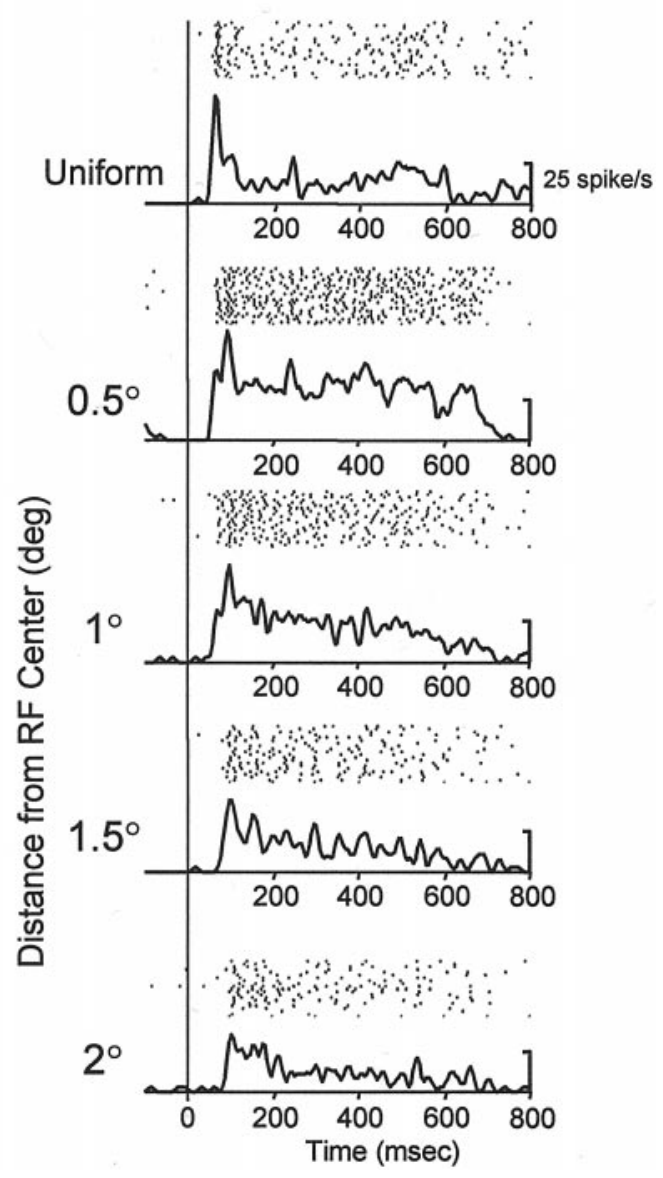

to, the RF borders. Second, the number of neurons that responded significantly better to the orientation-defined figure than to the uniform texture was greatly reduced with figures $>2^{\circ}$. Of those V2 neurons that responded better to the orientationdefined figures (Table 2, row 3), all but one neuron responded to the texture surround when presented in isolation (Fig. 1E). Thus, V2 neurons responded to the texture stimuli in very much the same way as did those recorded in V1.

\section{Responses from the RF surround: V1 recordings}

In the previous sections, we described how neurons in V1 and V2 responded to texture elements within the RF under conditions in which the context of RF stimulation was defined by cues located outside the RF. Unexpectedly, we observed that the surround stimulus alone could often evoke a significant response (i.e., although there were no texture elements within the RF; see Fig. $8 A$ ). Even for surrounds with inner diameters of $4^{\circ}$, which were well beyond the border of the largest RF $\left(1^{\circ}\right), 44 \%$ of the V1 neurons responded to surround stimulation. This high percentage of neurons exhibiting extra-RF activation was surprising because, by definition, previous mapping with small patches of texture did not elicit a neuronal response at that distance (see Materials and Methods). To determine the spatial extent over which a surround stimulus alone could evoke a response, we systematically varied the distance of the inner border of the surround texture from the RF. Figure $8 B$ illustrates the response of a single V1 neuron, having a $1^{\circ} \mathrm{RF}$, to the surround stimulus presented at different distances from the RF. Although the average response of the neuron decreased as the inner boundary of the surround was presented at increasing distances from the RF, the surround stimulus elicited a significant response even when the inner boundary was $2^{\circ}$ from the RF center (i.e., clearly outside the RF). The raster plots demonstrate that the response to the surround stimulus was consistent from trial to trial for all stimulus conditions.

One characteristic feature of the response to the surround stimulus was that it occurred later in time relative to the response to direct RF stimulation. Figure $8 C$ illustrates the average response latency for this neuron plotted as a function of the distance of the surround stimulus from the RF center. An asterisk in this figure indicates that the latency of the response to the surround stimulus was significantly greater than was the latency of the response to the uniform texture ( $p<0.01$, one-tailed $t$ test). The latency of the response to the surround was significantly greater than was the latency to the uniform texture for distances from the RF center of $1.5^{\circ}$ or greater. Figure 9 illustrates the relationship between response latency and the distance of the inner boundary of the surround stimulus from the RF center for our sample of neurons that had RFs $1^{\circ}$ in diameter or smaller. The average latency of the response to the uniform texture was 64 msec (ranging from 40 to $100 \mathrm{msec}$; shown as the data point at 


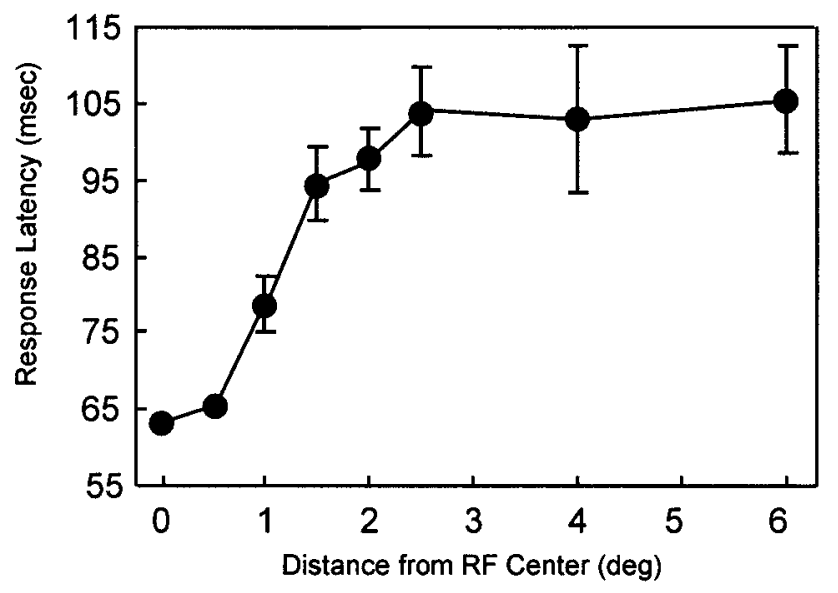

Figure 9. Average onset latency as a function of figure diameter. Data shown are for single and multiunit V1 sites with RF sizes $1^{\circ}$ in diameter or smaller that exhibited significant responses to the surround texture when presented alone. The number of neurons that contributed to the average for each figure size is given in Table 1. Error bars represent SEMs.

zero on the $x$-axis in Fig. 9). The positive slope of the curve in Figure 9 indicates that there was an abrupt increase in the average response latency when the surround boundary was $>0.5^{\circ}$ from the RF center. The average response latency increased to $\sim 105 \mathrm{msec}$ (range of 70-165 msec) as the distance between the surround and the RF center increased to $2.5^{\circ}$, beyond which the response latency was approximately constant. Although there was a limit to the distance from the RF for which a reliable response could be elicited by the surround stimulus, in 7 of 23 neurons it was possible to elicit significant responses with surrounds presented $6^{\circ}$ beyond the center of the RF. Table 1, column C, shows the number of neurons in our sample that contributed to the averages illustrated in Figure 9.

To investigate whether a complete figure formed by the surround texture was necessary to elicit the extra-RF response, we examined the responses of nine single neurons to partialsurround stimulation. For each neuron, we found that the response to the surround was not dependent on there being a complete figure (i.e., a "hole") defined by the texture surround. Figure 10 shows the response of a V1 neuron to different variations of the surround stimulus (black lines in graphs) compared with the response to the uniform texture (gray lines in graphs). The responses to partial surrounds were approximately equal in magnitude to the response elicited by the complete surround. Additionally, the same relative lag in the response latency $(\sim 30$ msec) to the complete surround, as compared with the uniform texture, was observed for the partial surrounds. Although the response of the neuron shown in Figure 10 did not show any anisotropy in spatial selectivity for the partial surrounds, three of the nine neurons did respond somewhat better to some partial surrounds than to others. However, for only one of these three neurons was the difference significant ( $t$ test, $p<0.05$ ). Taken together, the results demonstrate that the responses to the surround alone did not require the presence of a figure within the region of the $R F$.

\section{DISCUSSION}

Nearly all neurons studied responded similarly to an orientationdefined figure and to a uniform texture, except when the boundaries of the figure were within $1^{\circ}$ of the RF border. The limited spatial extent of the contextual modulation was not increased either at low stimulus contrast or when the animal was rewarded for detecting an orientation-defined figure. Thus, in agreement with their role in feature analysis on a very fine spatial scale, V1 neurons are very sensitive to the presence of textures and texture boundaries within or near their RF borders.

Unexpectedly, many V1 neurons responded to a surround texture located entirely outside the RF. More than $40 \%$ of neurons gave significant responses to the surround texture alone at distances $>1^{\circ}$ from the RF border, and some neurons even responded to the surround stimulus when its interior border was up to $5^{\circ}$ from the RF border. The response to the surround texture alone was much slower $(70-165 \mathrm{msec})$ than were responses to textures inside the RF (40-100 msec). Furthermore, the onset latencies for surround stimuli increased with their distance to the RF border. Although the typical stimulus used to stimulate the surround was a texture annulus, which might be interpreted to be a figural hole, we found similar results in a subpopulation of cells tested with surround stimuli that did not form a complete figure around the RF. Thus, although V1 neurons do receive significant information about texture stimuli located beyond their RF boundaries, their responses do not seem to depend on these stimuli forming figures.

\section{Comparison with previous studies}

The present results differ in several ways from studies of contextual interactions in V1 with orientation-defined figures. Under stimulus conditions and retinal eccentricities similar to those in the present study, Lamme (1995) reported that V1 neurons gave enhanced responses when the RF was located within the interior of an orientation-defined figure compared with a uniform background stimulus. In an extension of this work, Zipser et al. (1996) found that the enhanced responses to the orientation-defined figures were maintained for large figure sizes. Approximately $40 \%$ of V1 neurons showed significant enhancement for orientation-defined figures $3^{\circ}$ in diameter, and some neurons responded to figures as large as $8^{\circ}$ in diameter. It made little difference where the RF was located with respect to the figure borders, as long as it was in the interior of the figure rather than outside. By contrast, we found such enhancement only when the $\mathrm{RF}$ was inside small figures, close to the figure boundary. Even for small texture figures approximately the size of the RF, the magnitude of contextual effects was 20-30\% smaller, and the proportion of neurons exhibiting these effects was less than half the proportion reported by Zipser et al. (1996).

Recently, Lee et al. (1998) reported differences in the magnitude of enhancement effects for figural stimuli depending on the position of the RF within the figure. Specifically, responses to figural boundaries within the RF were approximately four times greater than were the enhancement effects observed when the RF was centered on the figure, consistent with the idea that V1 neurons respond primarily to local figure boundaries, as we found in the present study. However, unlike Lee et al., we found little or no enhancement for texture figures when the boundary was $>1^{\circ}$ from the RF border. Thus, our results suggest that V1 neurons mainly provide information about local texture boundaries, rather than the segregation of figures from the background.

What might account for these differences between the present and previous studies? One possibility is a difference in the behavioral response of the monkey. We recorded from neurons while the monkey was either passively fixating or performing a Go or No-Go detection task for texture-defined figures. The behavioral 
response in the latter task was a lever release while the eyes remained fixated on a fixation target. By contrast, in several of the previous studies (Lamme, 1995; Zipser et al., 1996; Lee et al., 1998) monkeys were rewarded to saccade to a luminous target that appeared at a random location after the presentation of the texture. It is therefore possible that preparation for a saccade leads to enhancement that is not found when the stimulus triggers a nonspatial motor response, such as in the detection task we used. However, another study found enhancement effects even when monkeys passively fixated a target and were not required to make a saccade to a figure (see Lamme et al., 1998). Whether or not task dependency turns out to explain some of the difference in results across studies, the important point is that the failure to find contextual modulation in some tasks argues against V1 playing a general role in figure-ground segmentation.

\section{The role of boundary contours}

Our finding that enhanced responses to orientation-defined figures diminished with distance from the figure boundary to the RF borders could be explained by at least two possible mechanisms. One is that the surround of the RF is suppressive, and the larger the figure covering the RF, the more the suppressive surround is stimulated. Alternatively, the texture boundaries of a figure could enhance the response to the texture elements contained within the RF, and this enhancement falls off with distance of the figure boundary from the RF. Excitatory inputs from the surround are supported by our finding of excitatory responses to a texture stimulus located entirely outside the RF. Thus, the close proximity of the figure boundary to the RF border was the likely cause of the contextual modulation observed with orientation-defined figures.

However, this mechanism, by itself, does not explain why $40 \%$ of the neurons responded to the surround stimulus alone presented $>2^{\circ}$ from the RF border, whereas the enhancement caused by the boundaries of the orientation-defined figure covering the RF was limited to $1^{\circ}$ of the RF border. One possibility is that the salience of the surround texture presented alone was much greater than that of the orientation-defined texture covering the RF. In addition, the enhanced response to an orientation-defined figure was necessarily measured as a modulation of the response to a texture stimulating the RF (i.e., a difference between two excitatory responses), whereas the response to the surround stimulus presented alone was measured in isolation. When the neuron is stimulated previously by texture elements inside the RF, additional excitatory inputs from the RF surround may not add linearly to the RF inputs.

\section{Long-latency responses from the surround}

The long-latency responses to the surround stimulus presented alone suggest a number of possible underlying mechanisms. Sceniak et al. (1999) found that the spatial extent of RF summation in V1 depended on stimulus contrast. The average area of spatial summation was more than two times greater with low-contrast stimuli than with high-contrast stimuli. The slight increase we observed in the figure modulation index for low-contrast orientation-defined figures compared with high-contrast figures is consistent with this explanation (see Fig. $4 B$ ). In view of these findings, our use of high-contrast stimuli to map the RF could have resulted in an underestimation of the summation area for stimulus conditions in which the surround texture was presented alone (i.e., no contrast in the RF). Although Sceniak et al. (1999) did not report latency data, the longer-latency responses we observed with the texture surrounds could be the result of contrast summation at the outer margins of the extended RF.
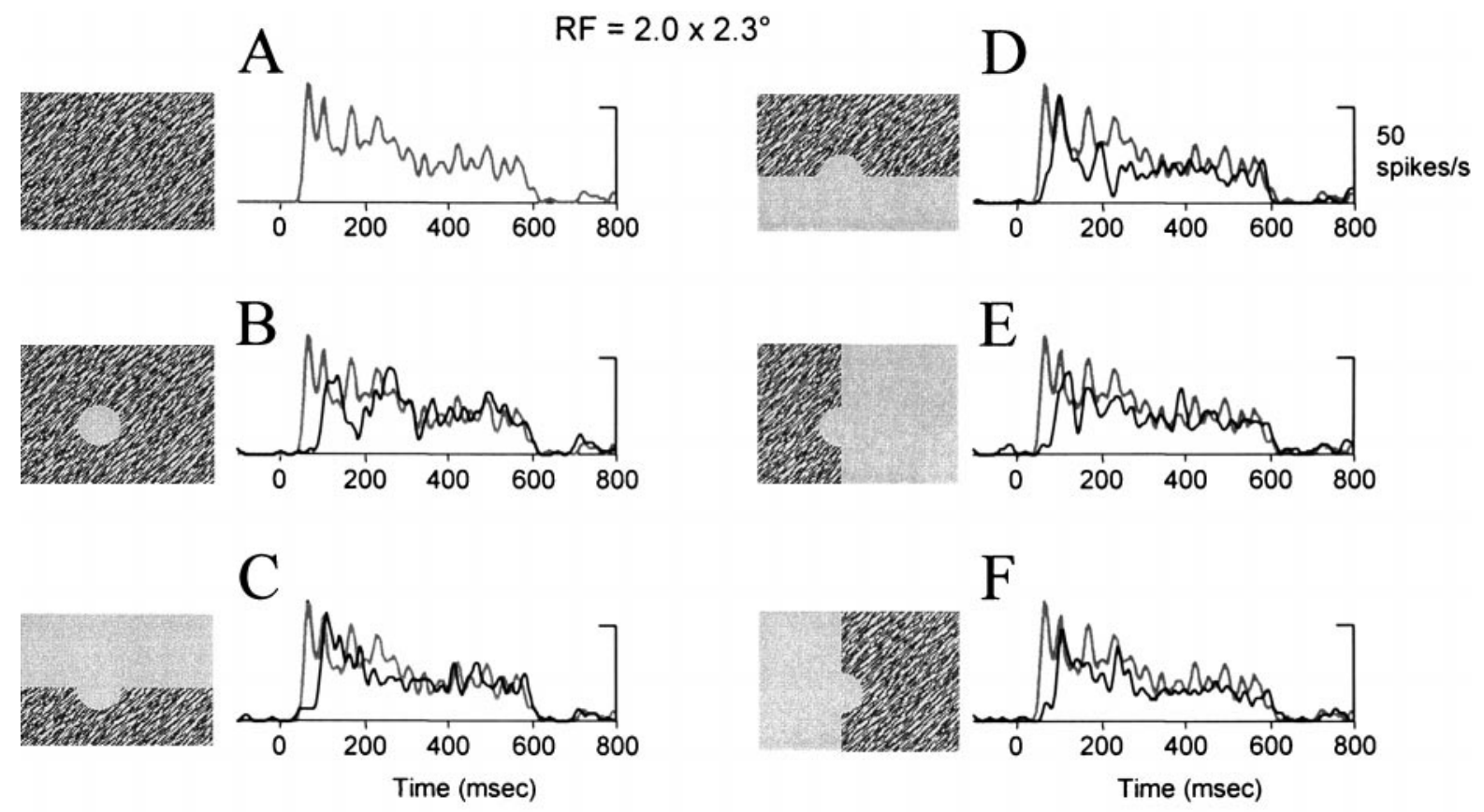

\section{DN110697.6:1}

Figure 10. Effects of partial field surround stimulation. $A$, The response of a single V1 neuron to the uniform texture. $B$, The response to the surround texture alone. $C-F$, Response of the neuron to partial surrounds. The black line in $B-F$ corresponds to the average response of the neuron to the stimulus configuration represented to the left. For comparison, the gray lines in $A-F$ indicate the average response to the uniform texture. The diameter of the figure defined by the surround texture was $5^{\circ}$. The size of the RF, as measured with small bar stimuli, was $2.0 \times 2.3^{\circ}$. 
Alternatively, it might simply take a longer time for neurons to receive inputs from locations far from the RF. This input could arise from feedback from higher cortical areas, from the propagation of signals within V1, or from a combination of both. The approximately linear increase in response latency with increasing stimulus distance from the RF (see Fig. 9) is not easily accounted for by a simple feedback mechanism. On the other hand, propagation of signals within V1 itself could account for the increasing latency with increasing distance to the RF. Optical imaging studies of the cortical point-spread function have shown that a focal visual stimulus evokes a wave of propagating activity in V1 that extends to an area up to 10 times larger than the retinotopic site of initiation (Grinvald et al., 1994; Das and Gilbert, 1995). In addition, Bringuier et al. (1999) reported large integration fields in cat V1 using intracellular recording and comparing response fields derived from action potentials and those from depolarizing postsynaptic potentials. Integration fields determined for postsynaptic potentials were up to five times larger than those mapped with spikes. Interestingly, the latency of the depolarizing potentials increased with the distance of the stimulus away from the center of the integration field. It is therefore conceivable that long-latency, extra-RF responses with surround textures are a suprathreshold manifestation of the large-scale spatial integration observed in these studies.

In conclusion, although the results of the present experiments reveal far-field contextual effects, they do not support the idea that V1 neurons extract figures per se from their background. Rather, contextual information at the level of V1 may contribute to figural representations in extrastriate visual areas. Monkeys with lesions of area V4 are impaired in tasks that rely on the perception of texture-defined figures (DeWeerd et al., 1996; Merigan, 1996), suggesting that extrastriate cortex plays an important role in the segmentation of figure and background. In addition, a recent human brain imaging study (Kastner et al., 2000) reported significantly more activity in the extrastriate visual areas V4 and TEO, but not in V1 or V2, in response to texturedefined figures relative to uniform textures.

\section{REFERENCES}

Allman J, Miezin F, McGuinness E (1985) Stimulus specific responses from beyond the classical receptive field: neurophysiological mechanisms for local-global comparisons in visual neurons. Annu Rev Neurosci 8:407-430.

Bringuier V, Chavane F, Glaeser L, Fregnac Y (1999) Horizontal propagation of visual activity in the synaptic integration field of area 17 neurons. Science 283:695-699.

Das A, Gilbert CD (1995) Long-range horizontal connections and their role in cortical reorganization revealed by optical recording of cat primary visual cortex. Nature 375:780-784.
DeWeerd P, Desimone R, Ungerleider LG (1996) Cue-dependent deficits in grating orientation discrimination after V4 lesions in macaques. Vis Neurosci 13:529-538.

Gallant JL, Van Essen DC, Nothdurft HC (1994) Two-dimensional and three-dimensional texture processing in visual cortex of the macaque monkey. In: Early vision and beyond (Papathomas TV, Chubb C, Gorea A, Kowler E, eds), pp 89-98. Cambridge, MA: Massachusetts Institute of Technology.

Gilbert CD, Wiesel TN (1990) The influence of contextual stimuli on the orientation selectivity of cells in primary visual cortex of the cat. Vision Res 30:1689-1701.

Grinvald A, Lieke EE, Frostig RD, Hildesheim R (1994) Cortical pointspread function and long-range lateral interactions revealed by realtime optical imaging of macaque monkey primary visual cortex. J Neurosci 14:2545-2568.

Kapadia MK, Ito M, Gilbert CD, Westheimer G (1995) Improvement in visual sensitivity by changes in local context: parallel studies in human observers and in V1 of alert monkeys. Neuron 15:843-856.

Kastner S, Nothdurft H, Pigarev IN (1997) Neuronal correlates of popout in cat striate cortex. Vision Res 37:371-376.

Kastner S, De Weerd P, Ungerleider LG (2000) Texture segregation in the human visual cortex: a functional MRI study. J Neurophysiol 83:2453-2457.

Knierim JJ, Van Essen DC (1992) Neuronal responses to static texture patterns in area V1 of the alert macaque monkey. J Neurophysiol 67:961-980.

Lamme VAF (1995) The neurophysiology of figure-ground segregation in primary visual cortex. J Neurosci 15:1605-1615.

Lamme VAF, Zipser K, Spekreijse H (1998) Figure-ground activity in primary visual cortex is suppressed by anesthesia. Proc Natl Acad Sci USA 95:3263-3268.

Lee TS, Mumford D, Romero R, Lamme VAF (1998) The role of primary visual cortex in higher level vision. Vision Res 38:2429-2454.

Li W, Thier P, Wehrhahn C (2000) Contextual influence of orientation discrimination of humans and responses of neurons in V1 of alert monkeys. J Neurophysiol 83:941-954.

MacEvoy SP, Kim W, Paradiso MA (1998) Integration of surface information in primary visual cortex. Nat Neurosci 1:616-620.

Maffei L, Fiorentini A (1976) The unresponsive regions of visual cortical receptive fields. Vision Res 16:1131-1139.

Maunsell JHR, Gibson JR (1992) Visual response latencies in striate cortex of the macaque monkey. J Neurophysiol 68:1332-1344.

Merigan W (1996) Basic visual capacities and shape discrimination after lesions of extrastriate area V4 in macaques. Vis Neurosci 13:51-60.

Miller EK, Li L, Desimone R (1993) Activity of neurons in the anterior inferior temporal cortex during a short-term memory task. J Neurosci 13:1460-1478.

Nelson JI, Frost BJ (1978) Orientation-selective inhibition from beyond the classic receptive field. Brain Res 139:359-365.

Nelson JI, Frost BJ (1985) Intracortical facilitation among co-oriented, co-axially aligned simple cells in cat striate cortex. Exp Brain Res 61:54-61.

Polat U, Mizobe K, Pettet MW, Kasamatsu T, Norcia AM (1998) Collinear stimuli regulate visual responses depending on cell's contrast threshold. Nature 391:580-584.

Rossi AF, Rittenhouse CD, Paradiso MA (1996) The representation of brightness in primary visual cortex. Science 273:1104-1107.

Sceniak MP, Ringach DL, Hawken MJ, Shapley R (1999) Contrast's effect on spatial summation by macaque V1 neurons. Nat Neurosci 2:733-739.

Zipser K, Lamme VAF, Schiller PH (1996) Contextual modulation in primary visual cortex. J Neurosci 16:7376-7389. 